\title{
Prof. Chun Chen: preoperative planning is essential in a segmental resection
}

Submitted Nov 25, 2018. Accepted for publication Dec 06, 2018.

doi: $10.21037 /$ jtd.2018.12.34

View this article at: http://dx.doi.org/10.21037/jtd.2018.12.34

\section{Editor's note}

The third Ruijin Chest International Forum was held in Shanghai on November 16th, 2018. The forum was based on the new developments in the minimally invasive treatment of thoracic tumors. It focused on the hot topics in the field of thoracic surgery, and it invited top experts from home and abroad to share their experiences.

After the meeting, professor Chun Chen (Figure 1), the deputy President of the Union Hospital of Fujian Medical University delivered a speech entitled "The importance of segmentectomy planning". He pointed out that preoperative simulation planning has three essential functions. One is to understand the anatomical variation fully, avoid the damage of essential structures, and then the doctors can develop individualized surgical plans. The second is to simulate the scope of surgery, determine the surgical margins, and determine the reasonable lung segments to resect. Third, uncooperating these two, it can reduce unnecessary exploration time, reduce trauma, and shorten operation time. During the meeting, AME editors interviewed Professor Chun Chen on the skills of segmentectomy, the status of preoperative planning, and prevention of perioperative complications.

\section{Expert introduction}

Chun Chen, MD, Department of Thoracic Surgery, Fujian Medical University Union Hospital, Fuzhou, Fujian, China.

Doctor Chen obtained his Medical Doctor degree from Fujian Medical University in 2007 and now is the director of Thoracic Surgery Department, Fujian Medical University Union Hospital, Fujian, China. His primary researches are focused on general thoracic surgery, especially minimally invasive thoracoscopic surgery.

Doctor Chen has made a great success in video-assisted

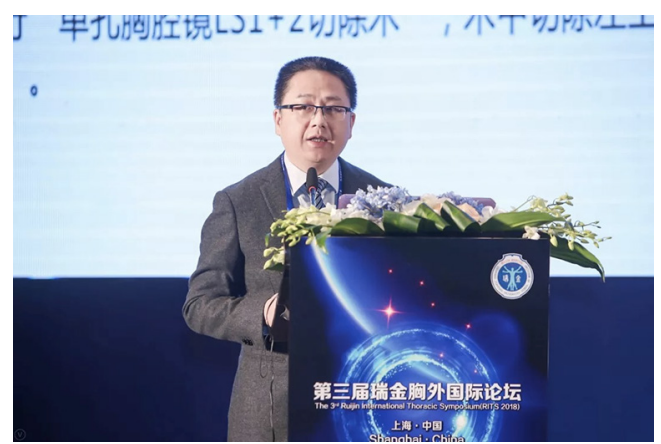

Figure 1 Professor Chun Chen.

thoracic surgery (VATS). He successfully introduced single-port VATS (SLVATS) anatomic segmentectomy in Fujian province. Moreover, he made some modification in SLVATS, such as the introduction of IQQA system and application of ultrasonic devices to divide the intersegmental plane, which increases the safety and accuracy of segmentectomy.

\section{Interview questions}

(I) You mentioned in your speech that preoperative simulation planning could reduce the damage, reduce the difficulty of surgery, and improve the accuracy of surgery. So, what is the current status for the preoperative planning application related to the usage of technology? What are the difficulties?

(II) What are the advantages of the usage of robotics for a more accurate pulmonary segmentectomy?

(III) What is your experience with the accurate identification of the pulmonary veins and the precise separation of the planes between the segments?

(IV) In your opinion, what is needed for the prevention of complications during the perioperative period of a 
thoracic surgery?

(

What experience have you accumulated in perioperative lung protection? What changes has this brought to the patient?

\section{Interview summary}

\section{Segmentectomy requires patience for fine work}

To perform a segmentectomy, you must have patience, persistence, and precision to achieve a high-quality operation. On the one hand, the blood vessels in the lungs are relatively thin, and the position is deep. It is easy for it to be damaged when dissecting, and must be carefully and patiently separated. If there are some minor damages in the process, compression and hemostasis are better than direct electrocoagulation. On the other hand, we are now using the ventilation discrimination method to separate the intersegment planes. It is essential to take time and be patient so that the doctor can ensure that the plane separation between the segments is more precise, so after the patient inhales pure oxygen, it is necessary to wait patiently for the boundary of the resection point to appear; this takes about ten minutes. When separating the plane between the segments, we generally use an ultrasonic scalpel, which has a good effect on the patient's cavity and can achieve a more precise peeling effect than the traditional electric hook.

\section{Preoperative planning projects might be included in bealth insurance}

In the future, preoperative planning is essential for pulmonary segmentectomy. However, due to the specific gaps in the hardware equipment, doctor's surgical technique and the operational concept of different levels of hospitals, the current pre-operative planning is not the same. Since July last year, we have started pre-planning lectures throughout the country. After more than a year, we can see that the acceptance of pre-operative planning concepts and techniques is getting higher and higher. Soon, pre-planning related projects may be included in medical insurance and formally enter the clinic.

The advent of the fourth generation of robots might be more conducive to the operation of the pulmonary segmentectomy

The flexible robotic arm of the surgical robot and the high-definition field of view enhance the accuracy of the lung segment surgery. Recently, the fourth-generation surgical robots appeared at the China International Import Expo, which has the advantages of less trauma and less bleeding and is more beneficial for segmental resection. However, there are currently only 73 surgical robots in the country. The experience of robotic segmentectomy is still concentrated on a few units and individuals. It is expected that robotic surgery will be further developed in the future.

\section{Perioperative complications prevention is a systematic project}

Preventing complications is an eternal topic in thoracic surgery. Complications such as anastomotic leakage and pulmonary infection can seriously affect the postoperative recovery of patients, and may even cause an increase in the mortality rate. Postoperative hospital stay days are an essential criterion for measuring the management level of the perioperative period. We reduced the postoperative hospital stay from 10.3 to 8.4 days through the management of esophageal cancer patients. The number of patients with postoperative complications and delayed discharge was minimal, and the incidence of anastomotic leakage was almost zero.

In the perioperative lung protection, multiple factors should be considered comprehensively. Before the operation, we encourage patients to check their sputum and carry out aerosol inhalation treatment. They should also gargle 3 days before surgery to maintain oral hygiene; minimize the operation time during surgery, reduce the damage to the airway, and pay attention to heat preservation, each step of the operation must be managed correctly. To avoid air leaks, with inhalation, encourage the patients to cough and to check their sputum after surgery, and get out of bed as soon as possible. In the long-term, patients should also be observed for pulmonary infection, pleural effusion, and cardiovascular complications due to various reasons after surgery. Perioperative complications prevention is a systematic project, and each ring is not to be lost.

\section{Acknowledgements}

The authors would like to thank Prof. Chun Chen for 
participating in the interview.

\section{Footnote}

Conflicts of Interest: The authors have no conflicts of interest to declare.

(Interview Editors: Chen Gao, Jeremy Dean Chapnick, JTD, jtd@amepc.org)

Cite this article as: Gao C, Chapnick JD. Prof. Chun Chen: preoperative planning is essential in a segmental resection. J Thorac Dis 2018;10(12):E850-E852. doi: 10.21037/ jtd.2018.12.34 